\title{
O Processo Histórico dos Métodos de Contagem como estratégia de ensino e aprendizagem
}

\section{The Historical Process of Counting Methods as a teaching and learning strategy}

\author{
Nélio Santos Nahum ${ }^{1 *}$, Sebastião Martins Siqueira Cordeiro ${ }^{1}$, Ataíde das Chagas Dias ${ }^{1}$, \\ Antonio Maia de Jesus Chaves Neto ${ }^{1}$, José Francisco da Silva Costa ${ }^{1}$
}

\begin{abstract}
RESUMO
Este trabalho de pesquisa aborda os métodos de contagem estudados no Ensino Médio. Com ênfase numa pesquisa bibliográfica em livros e artigos científicos, e pautada no processo histórico buscando mostrar a trajetória dos problemas que deram inicio aos métodos de contagem. A motivação para propor esta abordagem surgiu das da possibilidade de mostrar no contexto da sala de aula a relevância do aluno compreender conteúdos matemáticos a partir da história, destacando os matemáticos que desenvolveram a partir de situações advindas até mesmo do cotidiano, situações que requeriam uma interpretação matemática de um determinado problema real. Nesse sentido, busca-se investigar e compreender os métodos de contagem, partindo de uma proposta que direcionada a problemas a saber: Quadrados Mágicos (Lo-Shu), O Stomachion, Jogos de Azar; As Pontes de Königsberg e o Teorema das Quatro Cores. A pesquisa tem sua ênfase para o ensino de modo a motivar o aluno no entendimento do processo histórico que ocorreu para a evolução dos métodos de contagem. Conclui-se a pesquisa considerando que a ciência para ser bem compreendida necessita de duas principais linhas, a primeira sendo a História evolutiva e no formalismo de teoremas e axiomas que resultam da investigação e análises de situações problemas que asseguram o caráter cientifico.
\end{abstract}

Palavras-chaves: Processo Histórico; Métodos de Contagem; Ensino.

\begin{abstract}
This research work addresses the counting methods studied in high school. With an emphasis on bibliographical research in books and scientific articles, and based on the historical process, seeking to show the trajectory of the problems that gave rise to counting methods. The motivation to propose this approach arose from the possibility of showing, in the classroom context, the relevance of the student to understand mathematical content from history, highlighting the mathematicians who developed from situations arising even from everyday life, situations that required an interpretation mathematics of a given real problem. In this sense, we seek to investigate and understand the counting methods, starting from a proposal that addresses problems such as: Magic Squares (Lo-Shu), The Stomachion, Games of Chance; Königsberg Bridges and the Four Colors Theorem. The research has its emphasis on teaching in order to motivate the student to understand the historical process that occurred for the evolution of counting methods. The research concludes considering that science to be well understood needs two main lines, the
\end{abstract}

\footnotetext{
${ }^{1}$ Universidade Federal do Pará

1*E-mail: neliosnahum@yahoo.com.br
} 
first being the evolutionary history and the formalism of theorems and axioms that result from the investigation and analysis of problem situations that ensure the scientific character.

Keywords: Historical Process; Counting Methods;teaching.

\section{Introdução}

Na década de 70, em uma publicação intitulada Prelúdio à Análise Combinatória, Bachx et al (1975) menciona o estudo de análise combinatória como um sério obstáculo aos alunos do colegial (atualmente Ensino Médio) e aponta como possíveis causas o estudo restrito a definições e fórmulas, que segundo ele, conduzem o discente a um trabalho educacional mecânico que exclui a compreensão do que estão fazendo.

Em estudos recentes, Carvalho (2017), trata este tema como um paradoxo ${ }^{2}$, pois são em muitos casos considerados difíceis por alunos e professores, apesar de fazerem uso de técnicas elementares e atribui como possível causa o fato de que, diferentemente do que ocorre com outros assuntos da matemática, em que o ensino baseia-se em aplicações diretas de fórmulas e repetição de exemplos-modelos, problemas de contagem exigem o pensar, o entendimento da situação representada, indo além de uma aplicação direta de fórmulas. Situações relacionadas à contagem são frequentes no cotidiano e podem ser verificadas, por exemplo, quando se pensa nas possibilidades de combinação de roupas, no planejamento de pratos em cardápios ou de possíveis escolhas de números em um jogo de loteria.

Sobre o aspecto histórico o processo de contar provavelmente tem seu início, através do método de correspondência biunívoca, o ponto de partida se deu quando o homem desenvolveu a capacidade de comparar e relacionar conjuntos de objetos, estabelecendo entre eles uma correspondência. Para isso, fazia-se uso de partes do corpo, como os dedos das mãos ou dos pés, estes eram os métodos mais naturais de contagem, além destes, pedregulhos, conchas ou grãos, além de marcas no chão, na areia, em ossos ou madeira, poderiam ser utilizados para quantificar e estabelecer as relações necessárias, tais procedimentos explicam muito métodos de contagem que ainda hoje se utilizam (EVES, 2011).

\footnotetext{
${ }^{2}$ Paradoxo: o que é ou parece contrário ao senso comum.
} 
Os processos de contagem evoluem com o surgimento da análise combinatória, ou simplesmente combinatória em que Morgado et al (2016), a define, como a parte da matemática que analisa estruturas e relações discretas, tendo como objeto de estudo: demonstrar a existência, contar ou classificar os subconjuntos de elementos de um conjunto finito dado, que satisfazem certas condições. A aplicação de seus métodos, permitem resolver problemas com ênfase na escolha, arrumação e contagem dos elementos de um conjunto.

A BNCC apresenta habilidades que orientam a construção de novos métodos e práticas, de contagem, possibilitando a utilização dos conhecimentos prévios dos alunos trazidos para a sala de aula (AUSUBEL, 1973) na condição de ferramentas pedagógicas. Tendo em vista estas orientações, e como complemento do desenvolvimento deste trabalho, utiliza-se um um processo histórico dos métodos de contagem, destacando quatro (4) problemas que representaram a evolução matemática de todo um desenvolvimento desse contexto.

Assim sendo, a pesquisa procura direcionar o estudo apontando condições de se compreender a temática apontando como objetivo geral o processo histórico dos métodos de contagem como estratégia de ensino e aprendizagem o que conduz a uma metodologia de ensino que o professor pode utilizar na sala de aula como uma motivação para que o aluno venha adquirir um maior interesse por um determinado conteúdo matemático, possibilitando uma maior compreensão a partir desse processo histórico.

Quanto a essa motivação e justificativa da pesquisa, pode-se considerar o que surgiram com a necessidade de apontar uma alternativa metodológica que venha buscar uma aprendizagem em que o aluno consiga adentrar no processo histórico a fim de entender como a Matemática, a exemplo, o método de contagem, resultou na teoria que hoje se apresenta através de formalismo, axiomas e teoremas que foram surgidos a partir de situações de problema que são descritos ao longo dessa pesquisa. 


\title{
2-CONTEXTO HISTÓRICO DOS MÉTODOS DE CONTAGEM
}

Este tópico faz um resumo histórico dos métodos de contagem ${ }^{3}$. Ao contextualizar o modo de vida dos povos primitivos, aborda-se os primeiros indícios de contagem primitiva. Segue-se com a origem e evolução da combinatória, abordam-se os intrigantes problemas históricos, tais como: os quadrados mágicos, o Stomachion, as pontes de Königsberg (problema das sete pontes) e o Teorema das quatro cores, que contribuíram para a evolução dos métodos de contagem.

\subsection{Origens dos Métodos de Contagem}

Segundo Eves (2011), os métodos de contagem originaram-se muito antes da escrita ou mesmo da própria civilização, havendo pouco registro específico para análise. Sabe- se que esses processos são a base para o desenvolvimento de métodos matemáticos complexos, e sua compreensão é de fundamental importância para a história da matemática.

\begin{abstract}
O conceito de número e o processo de contar desenvolveram-se tão antes dos primeiros registros históricos (há evidencias arqueológicas de que o homem, já há uns 50.000 anos, era capaz de contar) que a maneira como ocorreram e largamente conjectural. Não e difícil, porém, imaginar como isso provavelmente se deu. E razoável admitir que a espécie humana, mesmo nas épocas mais primitivas, tinha algum senso numérico, pelo menos ao ponto de reconhecer mais e menos quando se acrescentavam ou retiravam alguns objetos de uma coleção pequena, pois há estudos que mostram que alguns animais são dotados desse senso. (EVES, 2011, p.25)
\end{abstract}

Do citado, pode-se considerar as habilidades naturais dos seres humanos de pensar em noções quantitativas básicas: muito e pouco, grande e pequeno, rápido e lento. Desta forma faz sentido considerar que povos primitivos tivessem certo sentido de números, por exemplo, ao adicionar ou remover objetos de uma pequena coleção.

Segundo Eves (2011), o processo de contar provavelmente tem seu início, através do método de correspondência biunívoca, o ponto de partida se deu quando o homem desenvolveu a capacidade de comparar e relacionar conjuntos de objetos, estabelecendo entre eles uma correspondência. Para estabelecer tais correspondências ele fazia uso de partes do corpo, como os dedos das mãos ou dos pés, que eram os métodos mais naturais

\footnotetext{
${ }^{3}$ Contagem: Ação de contar, de calcular, de avaliar algo. [Matemática] Indicação exata do número de elementos de um conjunto.(Aurélio, https://www.dicio.com.br/contagem/).
} 
de contagem, além destes, pedregulhos, conchas ou grãos, além de marcas no chão, na areia, em ossos ou madeira, poderiam ser utilizados para quantificar e estabelecer as relações necessárias.

Sobre o seu desenvolvimento Eves (2011), afirma que se deu de acordo com a evolução gradual da sociedade. Cita-se como exemplo: que as pessoas realizavam trocas e precisavam registrar a parcela de caça de cada família, ambas as atividades envolvem a ideia de contar, o que pode ser considerado o ponto de partida para o desenvolvimento de diversas ideias científicas. A evolução de formas mais primitivas para a vida em sociedade, criou a necessidade de um pensamento numérico aprimorado.

\subsection{Surgimento e Evolução da Combinatória.}

Ao abordar os aspectos históricos dos métodos de contagem, deve-se considerar o surgimento da combinatória, parte da matemática que estuda os problemas de contagem. Em relação a sua origem, cada autor discutido nesse trabalho apresenta o seu ponto de vista. Morgado et al (2016), considera o binômio $(1+x)^{n}$ como um dos primeiros problemas relacionados à combinatória. EVES (2011), credita tal feito a um diagrama numérico conhecido como lo-shu, o mais antigo exemplo de quadrado mágico. Tavares (2005) atribui o início do estudo de combinatória, a Arquimedes (287 a.C - 212 a.C), por meio do Stomachion.

A seguir abordam-se esses três pontos de vista, além das contribuições à combinatória, advindas dos jogos de azar e dos problemas históricos intitulados: as pontes de Königsberg (problema das sete pontes) e o Teorema das quatro cores.

\subsubsection{O Binômio $(1+x)^{\mathrm{n}}$.}

Morgado et al (2016), aponta o desenvolvimento do binômio $(1+x)^{n}$ como um dos primeiros problemas estudados ligados ao estudo de combinatória. Citando haver registro nos Elementos de Euclides ${ }^{4}$, em torno de 300 a.C. O binômio possui relação direta

\footnotetext{
${ }^{4}$ Elementos de Euclides: A obra de Euclides, escrita em torno de 300 a. C é composta de 13 livros ou capítulos e reúne os conhecimentos de geometria, álgebra e aritmética.
} 
como o Triângulo de Pascal $^{5}$ e era conhecido por Shih-Chieh, na China, (em torno de 1300) e antes disso pelos hindus e árabes. Afirma também que o matemático hindu Báskhara (1114 - 1185), sabia calcular o número de permutações, combinações e de arranjos de $n$ objetos.

\subsubsection{Quadrados Mágicos (Lo-Shu).}

Segundo EVES (2011), um dos clássicos matemáticos chineses mais antigos é o I - King ou Livro das Permutações. Nele aparece um diagrama numérico conhecido como Lo-Shu. Trata-se do exemplo conhecido mais antigo de quadrado mágico que segundo a lenda o primeiro a vê-lo foi o imperador Yu, por volta de 2200 a.C., decorando a carapaça de uma tartaruga divina que lhe apareceu as margens do rio Amarelo. Conforme representado na Figura (Figura 1). É um arranjo quadrado de numerais expressos por nós em cordas; nós pretos para números pares e brancos para números impares.

Figura 1- Representação do lo-shu

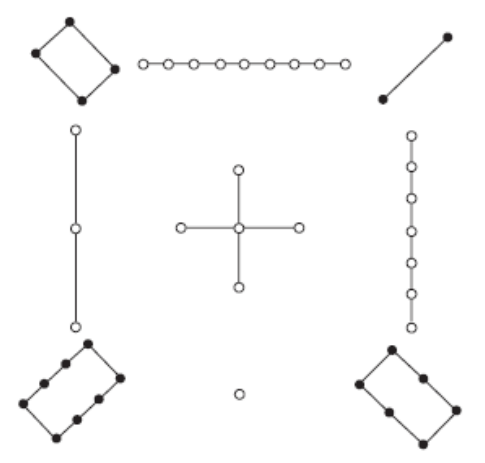

Fonte: Eves (2011, p.269).

Um quadrado mágico é um grupo ordenado de números $1,2,3, \ldots . ., \mathrm{n}^{2}$, distintos, dispostos de forma que cada linha, coluna ou diagonal deste quadrado possua a mesma soma, chamada constante mágica do quadrado, conforme mostra a figura (Figura 2). O quadrado mágico se diz normal se os $\mathrm{n}^{2}$ números que o formam são os $\mathrm{n}^{2}$ primeiros números inteiros positivos.

\footnotetext{
5 Triângulo de Pascal: Triângulo Aritmético de Tartaglia-Pascal (ou simplesmente Triângulo de Pascal), utilizado para calcular os coeficientes do desenvolvimento de potências do tipo $(x+y)^{n}$, com $x$ e $y$ reais e $n$ inteiro positivo.
} 
Figura 2 - Representação do quadrado mágico.

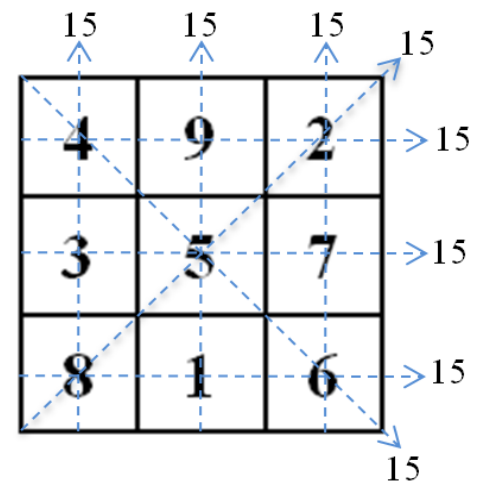

Fonte: acervo do autor.

Santinho (2006) cita que os chineses acreditavam que os quadrados mágicos possuíssem atributos místicos, e que simbolizavam os princípios básicos que formavam o universo, o número 5 representava a Terra e ao seu redor estão distribuídos os quatro elementos principais, a água 1 e 6 , o fogo 2 e 7 , a madeira 3 e 8 e os metais 4 e 9 . Acreditase que além do lado místico, despertaram também interesse em alguns matemáticos, como: Bernard Frénicle de Bessy (1602-1675), Claude- Gaspar Bachet (1581-1638), Pierre Fermat (1601-1665) e Leonhard Euler( 1707-1783).

Do aspecto de construção dos quadrados mágicos. Andrade (1999), demonstra uma expressão para o cálculo de $\mathrm{M}$, que representa a soma dos números de qualquer linha, coluna ou qualquer diagonal do quadrado), a qual denominou de constante mágica. Para isso, basta observar que a soma das $\mathrm{n}$ linhas da matriz é igual a:

$$
M+M+\cdots+M=n M
$$

Pode-se reescrever (1) da seguinte maneira:

$$
1+2+3+\cdots+n^{2}=\frac{n^{2}\left(n^{2}+1\right)}{2}
$$

Para a soma de todas as $n$ linhas, tem-se:

$$
\mathrm{nM}=\frac{\mathrm{n}^{2}\left(\mathrm{n}^{2}+1\right)}{2}
$$

Chega-se a expressão:

$$
M=\frac{n\left(n^{2}+1\right)}{2}
$$

Para descobrir a forma geral de um quadrado mágico de ordem 3. Andrade (1999) constrói o quadrado, conforme figura (Figura 3): 
Figura 3 - Representação genérica do quadrado mágico.

\begin{tabular}{|c|c|c|}
\hline$a$ & $b$ & $c$ \\
\hline$d$ & $e$ & $f$ \\
\hline$g$ & $h$ & $i$ \\
\hline
\end{tabular}

Fonte: acervo do autor.

Resolvendo o sistema linear formado pelas igualdades das somas de linhas, colunas e diagonais, e escolhendo $a$ e $b$ como variáveis livres, obtém-se um quadrado mágico de terceira ordem como mostra a figura (Figura 4):

Figura 4 - Representação do quadrado mágico em função das variáveis a e b.

\begin{tabular}{|c|c|c|}
\hline$a$ & $b$ & $15-a-b$ \\
\hline $20-b-2 a$ & 5 & $b-10+2 a$ \\
\hline$-5+a+b$ & $10-b$ & $10-a$ \\
\hline
\end{tabular}

Fonte: Acervo do autor.

Pode parecer que existem inúmeros quadrados mágicos de terceira ordem, bastando atribuir valores inteiros às variáveis $a$ e $b$, mas isso deve levar em consideração que o valor obtido deve ser um número inteiro que não se repita no intervalo $\left[1, \mathrm{n}^{2}\right]$. Logo (a, b), só podem assumir os valores: $(2,7),(2,9),(4,3),(4,9),(6,1),(6,7),(8,1)$ e $(8,3)$, gerando os quadrados mostrados na figura (Figura 5): 
Figura 5 - Representação dos quadrados mágicos de terceira ordem.

\begin{tabular}{|l|l|l|}
\hline 2 & 7 & 6 \\
\hline 9 & 5 & 1 \\
\hline 4 & 3 & 8 \\
\hline
\end{tabular}

\begin{tabular}{|l|l|l|}
\hline 2 & 9 & 4 \\
\hline 7 & 5 & 3 \\
\hline 6 & 1 & 8 \\
\hline
\end{tabular}

\begin{tabular}{|l|l|l|}
\hline 4 & 3 & 8 \\
\hline 9 & 5 & 1 \\
\hline 2 & 7 & 6 \\
\hline
\end{tabular}

\begin{tabular}{|l|l|l|}
\hline 4 & 9 & 2 \\
\hline 3 & 5 & 7 \\
\hline 8 & 1 & 6 \\
\hline
\end{tabular}

\begin{tabular}{|l|l|l|}
\hline 6 & 1 & 8 \\
\hline 7 & 5 & 3 \\
\hline 2 & 9 & 4 \\
\hline
\end{tabular}

\begin{tabular}{|l|l|l|}
\hline 6 & 7 & 2 \\
\hline 1 & 5 & 9 \\
\hline 8 & 3 & 4 \\
\hline
\end{tabular}

\begin{tabular}{|l|l|l|}
\hline 8 & 1 & 6 \\
\hline 3 & 5 & 7 \\
\hline 4 & 9 & 2 \\
\hline
\end{tabular}

\begin{tabular}{|l|l|l|}
\hline 8 & 3 & 4 \\
\hline 1 & 5 & 9 \\
\hline 6 & 7 & 2 \\
\hline
\end{tabular}

Fonte: Acervo do autor.

Observa-se que cada um dos oito quadrados, pode ser obtido de qualquer outro por troca de linha, troca de coluna ou transposição de matriz. Nesse caso, diz-se que esses quadrados são iguais e que existe apenas um quadrado mágico de ordem 3. De modo geral, o cálculo do número de quadrado mágico de uma determinada ordem é um problema não resolvido. Sabe-se apenas que as quantidades de quadrados mágicos de ordens menores do que 6 conforme tabela (Tabela 1) a seguir:

Tabela 1 - Quantidades de quadrados mágicos.

\begin{tabular}{c|c}
\hline Ordem & Quantidades \\
\hline 3 & 1 \\
\hline 4 & 880 \\
\hline 5 & 275.305 .224 \\
\hline 6 & desconhecido. \\
\hline
\end{tabular}

Fonte: Acervo do autor.

Segundo Vazques (2004), o matemático francês Frénicle (1693) apresentou todos os 880 quadrados de ordem 4, e nesta mesma época seu compatriota, De La Loubère (1691) descreveu um método de construção de quadrados de ordem ímpar conhecido como método de fronteira. 


\subsubsection{O Stomachion.}

Tavares (2005) atribui-se o início do estudo de combinatória, a Arquimedes (287 a.C - 212 a.C), e isso deve-se a uma publicação que despertou grande interesse e curiosidade de grandes matemáticos da época e que ainda hoje é objeto de estudo. Tratase do Stomachion, que acreditava-se ser um jogo, semelhante ao Tangram ${ }^{6}$, que consiste em determinar de quantos modos poderiam ser reunidas 14 peças planas, de diferentes formatos e tamanhos, que quando encaixadas devem formar um quadrado, conforme mostra a figura (Figura 6).

Figura 6 - Representação do Stomachion

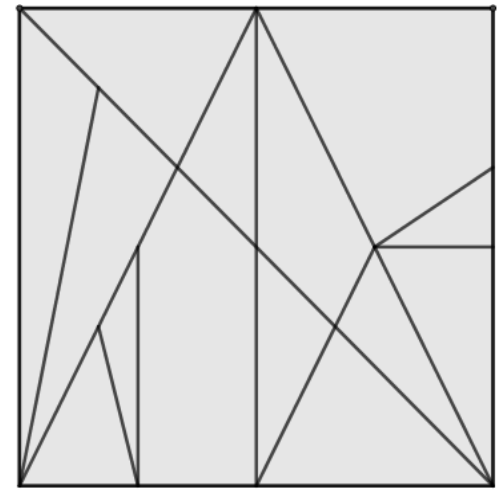

Fonte: Acervo do autor

O Stomachion, foi motivo de muita curiosidade e o questionamento era: como um simples jogo, foi capaz de despertar a atenção de uma das mais brilhantes mentes da história? Em dezembro de 2003, o jornal americano The New York Times publicou um artigo intitulado In Archimedes Puzzle, a New Eureka Moment, sobre os resultados da pesquisa do historiador de Matemática Reviel Netz, da Universidade de Stanford, Califórnia, em que ele afirma que o Stomachion não era um mero passatempo, mas um objeto executado por Arquimedes para fins de Análise Combinatória.

De maneira mais especifica, a conclusão de Netz é que Arquimedes desejava determinar de quantas formas distintas poderiam ser encaixadas as 14 peças para formar o quadrado. Desconsiderando as posições simétricas, chegou-se a 268 possibilidades, valor que não é possível verificar se Arquimedes encontrou.

\footnotetext{
${ }^{6}$ Tangram: Quebra cabeça geométrico, de origem chinesa.
} 
Segundo Tavares (2005), a resposta confirmada para essa questão pode ser 17.152 ou, desconsiderando as soluções simétricas, 268, o que nos parece mais razoável. Cita também que não está claro se Arquimedes obteve essa resposta, no entanto, como dito, há divergências sobre a origem da análise combinatória e neste caso atribui-se a Arquimedes, por meio do Stomachion.

\subsubsection{Jogos de Azar.}

No processo de desenvolvimento da combinatória Morgado et al (2016) atribui aos jogos de azar, importante contribuição. Jogadores buscavam maneiras seguras de ganhar em jogos de cartas, dados ou moedas. Entre eles, cita-se o cavalheiro De Meré, um homem que ficou na história como escritor. De Meré discutia com Pascal problemas relativos à probabilidade de ganhar em certos jogos de cartas e dados. Tavares (2005) menciona que um dos problemas era saber qual o número mínimo de vezes necessário para que se obtenha um "doble seis" (6 e 6) no lançamento de dois dados, um certo número de vezes.

Estava nascendo então a teoria das $\operatorname{probabilidades}^{7}$, campo fértil para o desenvolvimento da combinatória e objeto de estudo de matemáticos como: Blaise Pascal (1623-1662), Pierre de Fermat (1623-1662), Galileu Galileu (1564-1642), Jaime Bernoulli (1654-1705), Leonhard Euler (1710 -1761) e Laplace (1749-1827), se interessaram pelo assunto e contribuíram grandemente para seu desenvolvimento.

\subsubsection{As Pontes de Königsberg.}

Leonhard Euler, também enunciou e resolveu um problema que intrigava os matemáticos de sua época, conhecido como as pontes de Königsberg. Segundo Stewart

\footnotetext{
${ }^{7}$ Teoria das Probabilidades: É o ramo da matemática que cria, desenvolve e em geral pesquisa modelos que podem ser utilizados para estudar experimentos ou fenômenos aleatórios(MORGADO et al, 2016).
} 
(2009), este problema levou Euler a criar em 1735, um novo campo na matemática, denominado de teoria dos grafos ${ }^{8}$. Königsberg, que naquele tempo ficava na Prússia, é cortada pelo rio Pregelarme. Havia duas ilhas, ligadas às margens e entre elas próprias por sete pontes. O problema consistia em saber se era possível dar uma volta pela cidade passando uma, e somente uma vez por cada ponte, conforme mostra a figura (Figura 7), que mostra a representação feita por Euler.

Figura 7 - Desenho das Pontes de Königsberg feito por Euler.

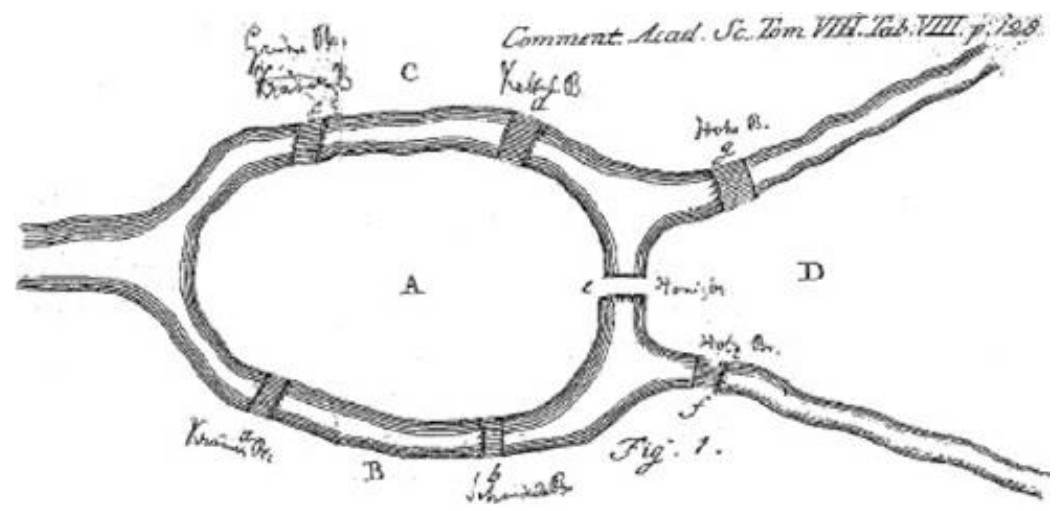

Fonte: Stewart (2009, p.60).

Euler provou não haver solução para o problema e apresentou de forma mais geral, critérios para que qualquer problema semelhante tenha solução, o que não se aplicava a este caso. Euler percebeu que a geometria precisa é irrelevante e o que importa é a forma como as coisas estão interligadas e assim foi capaz de reduzir o problema a uma rede de pontos unidos por linhas, conforme figura (Figura 8). Cada ponto correspondendo a uma porção de terra, e dois pontos estão unidos por linhas se houver uma ponte ligando as porções de terra correspondentes.

8 Teoria do Grafos: Como disciplina, surgiu no século XVIII, quando começou a ser estudada sistematicamente pelo influente e prolífico matemático suíço Leonhard Euler. Mas apenas no século XX ela chega à maturidade, passando de uma mera coleção de resultados desconexos a uma subárea estruturada da chamada Matemática Discreta (BENEVIDES, 2019). 
Figura 8 - Transformando as pontes de Königsberg numa rede.

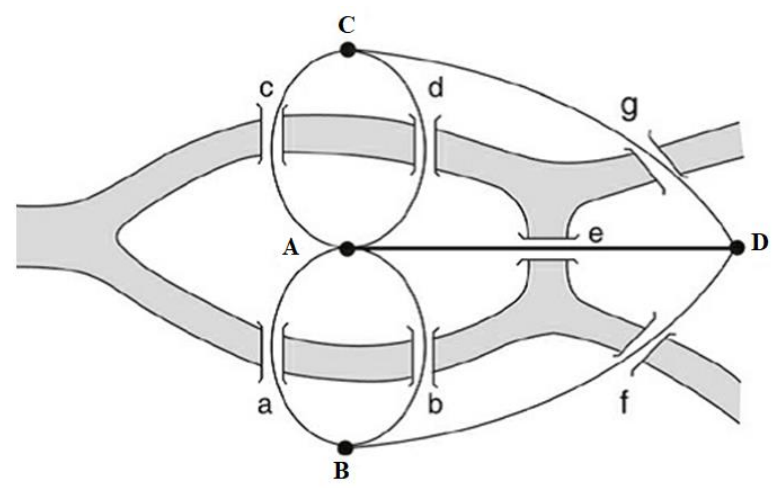

Fonte: Stewart (2009, p.61).

Portanto, tem-se os pontos A, B, C e D e as sete arestas a, b, c, d, e, f, g, uma para cada ponte. Isso permitiu reescrever a pergunta da seguinte maneira: É possível encontrar um caminho através da rede que passa por cada aresta exatamente uma vez?. Segundo Stewart (2009), para provar se tais problemas têm solução ou não, Euler distinguiu dois tipos de trajeto. Um aberto que começa e termina em pontos diferentes e o fechado que começa e termina no mesmo ponto. Concluiu que, nesta rede, nenhum dos dois tipos é possível.

\subsubsection{O Teorema das Quatro Cores.}

Outra importante contribuição à evolução da combinatória deve-se a um problema de enunciado simples, porém sua solução mostrou-se bastante complexa, trata-se do Teorema das Quatro Cores.

Stewart (2009) indica que o problema teve origem em 1852 com Francis Guthrie, estudante da University College, em Londres. Ao colorir o mapa dos condados ingleses, conforme figura (Figura 9), descobriu que conseguia fazer usando 4 cores, de modo que os condados adjacentes nunca tivessem a mesma cor. Ele escreveu uma carta para seu irmão Frederick, descrevendo o que ele pensava ser um simples enigma. Escreveu "Será que é possível colorir qualquer mapa no plano com 4 cores (ou menos) de modo que as regiões que possuem fronteiras comuns jamais tenham a mesma cor? ". 
Figura 9 - Colorindo os Condados da Inglaterra com quatro cores.
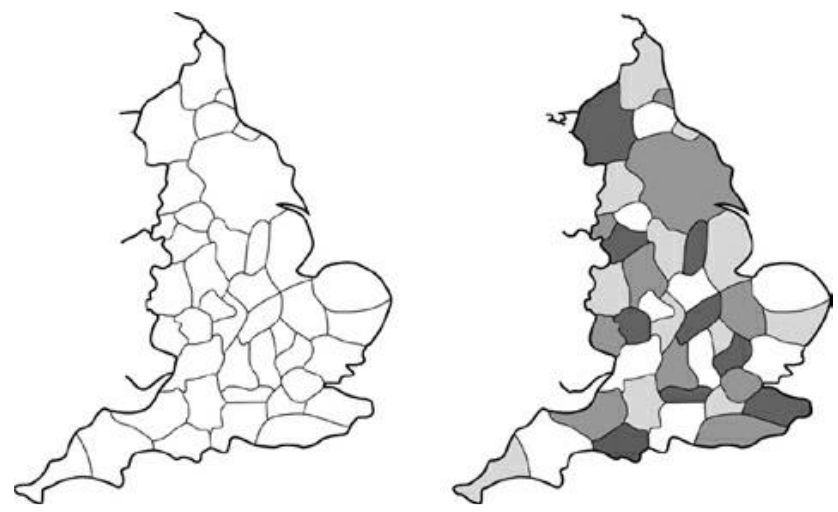

Fonte: Stewart ( 2009, p.20).

Frederick apresentou a conjectura de seu irmão a Augustus De Morgan", no entanto, soube-se que De Morgan não conseguiu, como confessou em outubro do mesmo ano em uma carta ao seu colega irlandês ainda mais famoso, Sir William Rowan Hamilton. De acordo com Santos (2008), De Morgan, obteve algum progresso. Inicialmente apontou que, se um mapa tem quatro áreas adjacentes duas a duas, ele precisa de quatro cores para ser colorido como mostra a figura (Figura 10).

Figura 10 - Mapa com quatro regiões adjacentes duas a duas.

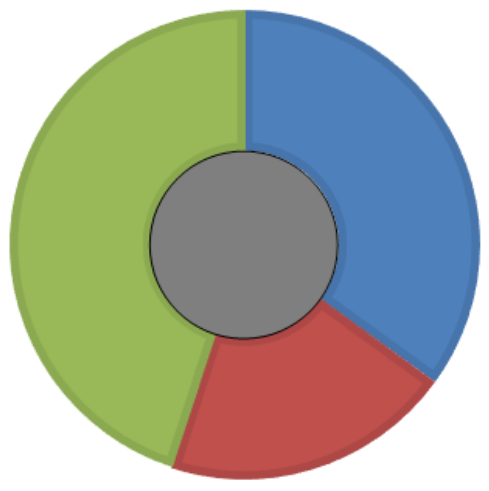

Fonte: Acervo do autor.

Em seguida, ele tentou determinar um mapa no qual cinco regiões fossem adjacentes, e provou que tal mapa não existe. Este fato não impede que existam mapas que necessitem de cinco cores. Um mapa que não possui quatro regiões adjacentes duas a duas pode exigir quatro cores para ser colorido, conforme figura (Figura 11). Este fato

\footnotetext{
${ }^{9}$ Augustus De Morgan (1806 - 1871): Matemático e lógico britânico.
} 
levou a conclusão que, mesmo o mapa que não possui cinco áreas adjacentes dois pode exigir cinco cores para ser colorido.

Figura 11 - Mapa que não possui quatro regiões adjacentes duas a duas.

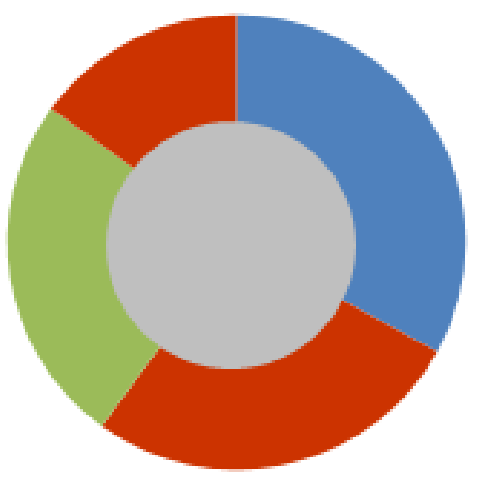

Fonte: Acervo do autor.

Depois de 1860, por mais ou menos 20 anos, o interesse dos matemáticos pelo problema diminuiu, não sendo discutido entre os matemáticos da época, no entanto não foi esquecido (PIMENTA, 2014). Em 1878, Arthur Cayley indagava na seção de Matemática da Royal Society se alguém já havia apresentado solução da Conjectura das Quatro Cores. Ninguém o resolvera, mas no ano seguinte Alfred Bray Kempe, um advogado e que tinha estudado no Trinity College de Cambridge, onde fora aluno de Cayley, publicou uma demonstração completa do Teorema das Quatro Cores no American Journal of Mathematics. A demonstração de Kempe foi estudada por vários matemáticos de renome e alguns deles fizeram sugestões para melhorar a demonstração. Portanto, em 1879, considerava-se definitivamente estabelecido o Teorema das Quatro Cores.

Segundo Sampaio (2004), em 1890, onze anos após a publicação de Kempe, Percy John Heawood, provou por meio de um contra-exemplo, que a demonstração de Kempe tinha um erro. Heawood foi além, demonstrou que cinco cores são suficientes para colorir qualquer mapa plano onde países de fronteiras comuns possuem cores diferentes.

Segundo Pimenta (2014). Durante 124 anos, muitas foram as tentativas de desenvolver métodos capazes de resolver o problema, mas somente, em 1976, com a 
ajuda de um IBM $^{10}$ 360, em Urbana (Illinois), Kenneth Appel e Wolfgang Haken apresentaram uma demonstração do Teorema das Quatro Cores. Quando a notícia desta façanha se espalhou, houve um enorme entusiasmo. Mas a euforia esfriou quando souberam que essa demonstração incluía mais de mil horas do uso de computadores de alta velocidade. A demonstração era demasiadamente longa para ser verificada sem o uso dos computadores e ainda havia a possibilidade dos computadores terem cometido alguma falha.

Stewart (2009), afirma que nos dias atuais, com computadores mais rápidos, podese repetir o processo em cerca de uma hora. Sabe-se que o Teorema das quatro cores é verdadeiro, o que responde à pergunta aparentemente inocente de Guthrie. E isso é uma conquista maravilhosa, mesmo que dependa de alguma ajuda de um computador, a construção de uma demonstração que não necessite do uso de computadores continua em aberto.

Estava dado um dos pontapés iniciais para a Teoria dos Grafos, fundada por Euler, Gustav Robert. Kirchhoff (1824-1887) e Arthur Cayley(1821-1895). Em 1834, Peter Gustav Lejeune Dirichlet (1805-1859) formulou o princípio das gavetas. Esse princípio aparentemente simples é um dos recursos mais utilizados para resolver problemas de combinatória.

É provável que nesse resumo histórico, outros matemáticos e suas contribuições não tenham sido mencionados, pois ao longo da história, muitos foram os que se dedicaram ao estudo da combinatória. Tavares (2005), refere-se aos avanços da combinatória na área computacional. Através de simulações em computadores, os pesquisadores dessa área podem fazer obter resultados que os permitem compreender e conjecturar problemas que são difíceis de resolver por métodos analíticos, levando à sua solução.

\footnotetext{
${ }^{10}$ IBM 360: IBM (International Business Machines Corporation) é uma empresa dos Estados Unidos voltada pra a área de informática. O IBM 360 constitui-se numa família de computadores de grande porte dedicado ao processamento de um volume enorme de informações, lançado Abril de 1964.
} 


\section{CONSIDERAÇÃO FINAL}

O texto descrito possibilitou nessa pesquisa que relacionou o processo histórico para o ensino dos métodos de contagem trouxe como propósito a valorização do pensar esse processo no contexto da sala de aula levando ainda a aprendizagem significativa dos alunos.

Para consolidar o estudo e ampliar o desenvolvido descrito, o professor deve articular a metodologia levando em conta esse processo histórico para que os alunos possam refletir acerca do processo de ensino e aprendizagem onde além de todo o desenvolvimento do formalismo matemático possui a chance de compreender melhor a ocorrência evolutiva da História o que pode o levar a investigar e testar suas práticas em situações problemas como os que foram apresentados ao longo dessa temática e com o auxílio do professor que pode inovar suas metodologias, criando estratégias que ajudem os alunos a entender a matemática sob esses dois aspectos histórico e analítico.

Dessa maneira o professor ao dar continuidade no seu trabalho educativo pode atrelar esse processo histórico, fazendo necessário que se aborde também o formalismo matemático, definições, axiomas, teoremas, demonstrações dentre outros, a fim de dar uma abrangência significativa o seu caráter cientifico.

\section{REFERÊNCIAS}

ANDRADE, L.A. Revista do Professor de Matemática Vol. 41: Mais sobre quadrados mágicos. Rio de Janeiro: SBM, 1999.

AUSUBEL, D. P. Alguns aspectos psicológicos de la estrutuctura del conocimiento. Buenos Aires: El Ateneo, 1973.

BACHX, A. de C.; POPPE, L. M. B.; TAVARES, R. N. O. Prelúdio à Análise

BENEVIDES, F.S, Introdução à Teoria dos Grafos - Parte 1. Disponível em : https://portaldaobmep.impa.br/index.php/modulo/ver?modulo=84. Acesso em 18/01/2021.

BRASIL. Base Nacional Comum Curricular (BNCC). Brasília: MEC, 2018.

CARVAlHO, P.C. Métodos de Contagem e Probabilidade. Rio de Janeiro: IMPA, 2017. 
Combinatória. São Paulo: Companhia Editora Nacional, 1975.

EVES, H. Introdução à História da Matemática - Tradução: Hygino H. Domingues 5a ed. Campinas, SP: Editora da UNICAMP, 2011.

MORGADO, A.C. et al. Análise Combinatória e Probabilidade. $10^{a}$ ed. Rio de Janeiro, SBM, 2016.

PIMENTA, M.M.D. História do Problema das Quatro Cores. Disponível em: http://clubes.obmep.org.br/blog/wp-content/uploads/2018/08/Quatro-Cores_2.pdf.

Acesso em 20/01/2021.

SAMPAIO, J.C.V. Quatro Cores e Matemática. II Bienal da Sociedade Brasileira de Matemática. Bahia, 2004.

SANTINHO, M.S; MACHADO, R.M. Os Fascinantes Quadrados Mágicos - III Bienal da Sociedade Brasileira de Matemática. Goiás: Anais, 2006.

SANTOS, R.A, Revista da Olimpíada IME n 7, p. 77-83. Goiás: UFG, 2008.

STEWART, I, Almanaque das Curiosidades Matemáticas - Tradução: Diego Alfaro. Rio de Janeiro: Zahar, 2009.

TAVARES, C.; BRITO, F.R.M: Revista do Professor de Matemática Vol. 57: Contando a História da Contagem. Rio de Janeiro: SBM, 2005.

VAZQUES, C. M. R.; NOGUTI, F. C. H. Análise Combinatória: alguns aspectos históricos e uma abordagem pedagógica. In: Encontro Nacional de Educação Matemática, VIII. Recife: Anais, 2004. 